\title{
Reverse Pharmacogenetic Modulation of the Nucleus Accumbens Reduces Ethanol Consumption in a Limited Access Paradigm
}

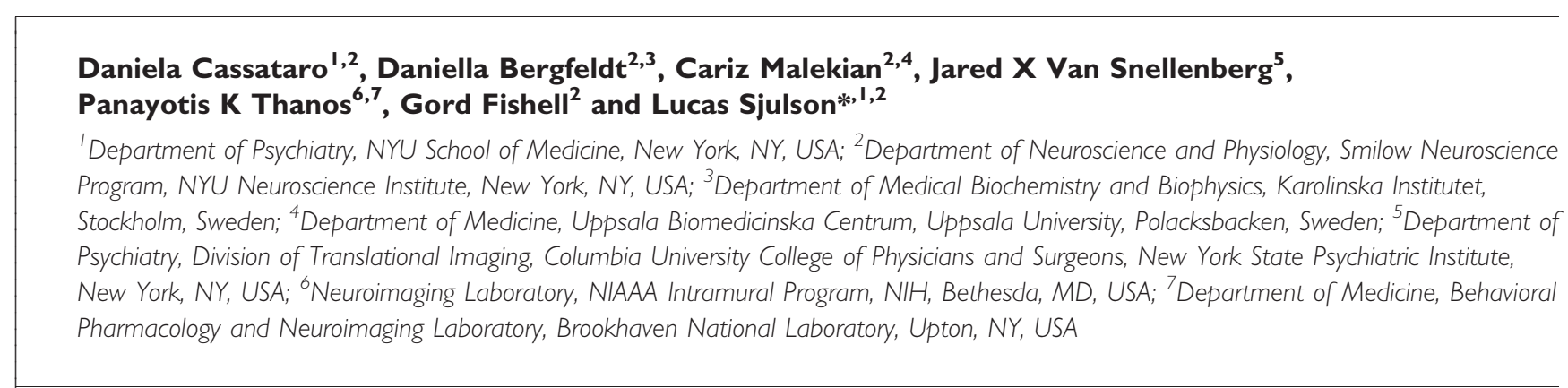

Bilateral stereotactic lesioning of the nucleus accumbens (NAc) core reduces relapse rates in alcohol-dependent patients but may cause irreversible cognitive deficits. Deep brain stimulation has similar effects but requires costly implanted hardware and regular surgical maintenance. Therefore, there is considerable interest in refining these approaches to develop reversible, minimally invasive treatments for alcohol dependence. Toward this end, we evaluated the feasibility of a reverse pharmacogenetic approach in a preclinical mouse model. We first assessed the predictive validity of a limited access ethanol consumption paradigm by confirming that electrolytic lesions of the NAc core decreased ethanol consumption, recapitulating the effects of similar lesions in humans. We then used this paradigm to test the effect of modulating activity in the NAc using the Designer Receptors Exclusively Activated by Designer Drugs (DREADDs) hM3Dq and hM4Di. We found that increasing activity with hM3Dq had no effect, but suppressing activity with hM4Di reduced alcohol consumption to a similar extent as lesioning without affecting consumption of water or sucrose. These results may represent early steps toward a novel neurosurgical treatment modality for alcohol dependence that is reversible and externally titratable, yet highly targetable and less invasive than current approaches such as lesioning or deep brain stimulation.

Neuropsychopharmacology (2014) 39, 283-290; doi:I0.1038/npp.20I3.I84; published online 23 October 2013

Keywords: alcohol dependence; reverse pharmacogenetics; nucleus accumbens; drinking in the dark; DREADDs

\section{INTRODUCTION}

Alcohol dependence is one of the leading public health problems in the developed world. In the United States alone, alcohol causes 80000 deaths annually (CDC, 2008) and costs an estimated $\$ 223.5$ billion per year (Bouchery et al, 2011). A large body of research has implicated a circuit containing the nucleus accumbens (NAc) and dopaminergic projections from the ventral tegmental area in alcohol dependence (Heinz et al, 2009; Koob and Volkow, 2010). However, no pharmacological treatment has been able to target this circuit and produce a substantial therapeutic benefit for alcohol dependence, primarily because pharmacological approaches lack the anatomical specificity to target specific

\footnotetext{
*Correspondence: Dr L Sjulson, Department of Psychiatry, NYU School of Medicine, Smilow 507, 522 Ist Avenue, New York, NY 10016, USA, Tel: + | 646528 9672, Fax: + | 2122639170

E-mail: Lucas.Sjulson@nyumc.org

Received II December 2012; revised 26 July 2013; accepted 29 July 2013; accepted article preview online I August 2013
}

circuits within the brain. Conversely, existing neurosurgical approaches possess high anatomical specificity but virtually no specificity at the level of cell types within the brain. This is particularly relevant in the context of the NAc, which contains multiple cell types, including a variety of interneuron populations (Tepper et al, 2010) and two major projection neuron populations known to have largely opposing functional roles (Lobo and Nestler, 2011). Nevertheless, bilateral stereotactic lesions of the NAc have been shown to reduce cravings and relapse in human subjects with alcohol dependence (Wu et al, 2010); however, this may cause permanent deficits in IQ and memory (He et al, 2008). Deep brain stimulators placed in the NAc show similar results (Heinze et al, 2009; Kuhn et al, 2011; Voges et al, 2012), but require chronic implantation of expensive indwelling hardware that requires regular surgical maintenance.

One strategy for overcoming these limitations is reverse pharmacogenetics. Unlike classical pharmacogenetics, which tailors a drug regimen to match a subject's genetic profile, reverse pharmacogenetics uses techniques from 
gene therapy to express drug targets ectopically, thus altering the subject's response to a given drug. The most successful example of this approach is the DREADDs or Designer Receptors Exclusively Activated by Designer Drugs (Armbruster et al, 2007; Rogan and Roth, 2011). These are G-protein-coupled receptors that respond only to clozapine-N-oxide (CNO), an inert compound with no known pharmacological activity. Two DREADDs are used commonly for neuromodulation: hM3Dq, which increases neuronal firing rates, and hM4Di, which inhibits firing. As DREADDs can be expressed ectopically in specific cell populations and activated by systemic administration of $\mathrm{CNO}$, they enable neuromodulation with simultaneous celltype specificity and anatomic specificity that is reversible and externally titratable.

DREADDs are currently impractical for use in humans because the non-native protein sequences may cause immune reactions and because humans, unlike mice, metabolize CNO to clozapine (Chang et al, 1998), a drug with numerous undesirable off-target effects. However, other reverse pharmacogenetic techniques applied to the NAc may one day prove to be a useful therapeutic strategy against drug and alcohol dependence. We thus aimed to evaluate the feasibility of this strategy in a preclinical rodent model. We first assessed the predictive validity of the 'Drinking in the Dark' (DID) limited access model (Rhodes et al, 2005) with respect to manipulations of the NAc by determining whether lesions of the NAc core would reduce alcohol consumption, as has been found in human subjects (Wu et al, 2010). We then used the DID model to test the hypothesis that reverse pharmacogenetic modulation of the NAc using DREADDs could reversibly decrease ethanol consumption to a similar extent.

\section{MATERIALS AND METHODS}

\section{Animals}

Male C57BL/6J mice were obtained from The Jackson Laboratory (Bar Harbor, ME). This strain of mice was chosen for its known propensity to consume pharmacologically relevant quantities of ethanol (Rodgers and McClearn, 1962). Mice were group-housed (five per cage) for 1 week before surgery in a temperature-controlled colony room with free access to food and water. Surgery was performed at 10-11 weeks of age. After surgery, mice were transferred to a second colony room with a 12-h reverse light/dark cycle for 2.5 weeks before behavioral testing; animals were individually housed starting 1 week before testing. The Institutional Animal Care and Use Committee at NYU approved all procedures.

\section{Electrolytic Lesions and Adeno-Associated Virus Vector (AAV) Injection}

Animals were anesthetized with isoflurane (5\% induction, $1 \%$ maintenance) and placed in a stereotactic head frame (Kopf Instruments, Tujunga, CA) on a temperaturecontrolled heating pad. For electrolytic lesions, an insulated tungsten electrode $(\sim 80 \mu \mathrm{m}$ diameter, Microprobes, Gaithersburg, MD) was used to place eight lesions at the corners of a cube $300 \mu \mathrm{m}$ wide centered around the stereotactic coordinates of the NAc relative to bregma: $1.4 \mathrm{~mm}$ anterior, $1.2 \mathrm{~mm}$ lateral, and $4.5 \mathrm{~mm}$ ventral. A $100-$ $\mu \mathrm{A}$ anodal current was delivered for $10 \mathrm{~s}$ at each lesion site. For sham-lesioned control animals, craniotomies were cut and the dura punctured. Stereotactic injection of AAV was performed using a similar procedure, except that a sharp glass pipette $(25-30 \mu \mathrm{m}$ diameter) was used to inject $300 \mathrm{nl}$ of AAV at a rate of $50 \mathrm{nl} / \mathrm{min}$. The pipette was left in place for $5 \mathrm{~min}$ after the injection to minimize backflow. AAV2/5DREADD constructs were obtained from the Lowell lab and packaged by the Penn Vector Core. AAV2/5-Cre and AAV2/ 5-EGFP stocks were obtained from the University of Iowa Gene Transfer Vector Core. AAV2/5-DREADD or AAV2/5-EGFP were mixed in a 2:1 ratio with AAV2/5-Cre before injection. All AAV stocks had titers in the range of $10^{12}-10^{13} \mathrm{vg} / \mathrm{ml}$.

\section{Verification of Lesion/AAV Injection}

Animals were given an overdose of sodium pentobarbital and perfused trancardially with $4 \%$ paraformaldehyde in ice-cold PBS. Brains were post-fixed for $1-2 \mathrm{~h}$, cryoprotected with $30 \%$ sucrose, and cryosectioned at $20 \mu \mathrm{m}$ thickness. Sections were immunostained for GFAP to detect electrolytic lesions or GFP/mCherry to detect AAV expression. Sections were photographed on an Olympus MVX10 microscope (Olympus, Center Valley, PA) with a Leica DFC340FX camera (Leica, Allendale, NJ). Images were scored for anatomical targeting accuracy by an investigator blinded to the identity of the animals.

\section{Ethanol/Sucrose/Water Consumption Procedures}

Ethanol consumption was monitored using two different DID protocols (Rhodes et al, 2005). Briefly, animals were individually housed on a reverse light/dark cycle. Starting at $3 \mathrm{~h}$ after lights out, the water bottle in each cage was replaced for several hours with a 10-ml graduated sipper tube filled with $20 \%$ ethanol (USP grade, Sigma-Aldrich), enabling measurement of ethanol consumption. For electrolytically lesioned animals, a 4-day DID protocol was used (Supplementary Figure 1A), which Rhodes et al found is sufficient to produce stable high levels of ethanol consumption. During the habituation phase on days 1-3, animals were given $2 \mathrm{~h}$ of daily access to $20 \%$ ethanol in tap water. On the testing day (day 4), animals were given $4 \mathrm{~h}$ of ethanol access and consumption was measured. For ethanol consumption in DREADD-expressing animals, a 13-day DID protocol was used (Supplementary Figure 1B). Animals were given $2 \mathrm{~h}$ of daily access to $20 \%$ ethanol during habituation on days 1-3, but during the testing phase on days $4-13$ they were given $4 \mathrm{~h}$ of daily access, which is compatible with the $5-10 \mathrm{~h}$ duration of DREADD action in vivo (Alexander et al, 2009; Ray et al, 2011).

To control for nonspecific effects of DREADD-mediated neuromodulation on natural rewards and oral fluid intake, an analogous DID protocol (Supplementary Figure 1C) was used in which animals were given $2 \mathrm{~h}$ of access to either a 1 or $5 \%$ sucrose solution for days 1-3, then tested on days 4-9 with $4 \mathrm{~h}$ of access to the same sucrose solution. Animals tested with $5 \%$ sucrose were also tested on days 10-15 with $4 \mathrm{~h}$ of access to tap water. Because this protocol includes two 
conditions, sucrose and water, fewer measurements for each condition are made in each mouse. We therefore increased the number of mice to achieve statistical power equivalent to the 13-day protocol. Between all testing sessions, the home cage water bottle was filled on alternating days with either tap water or $0.1 \mathrm{mg} / \mathrm{ml} \mathrm{CNO}$. Animals weighed 30$35 \mathrm{~g}$ and consumed approximately $3 \mathrm{ml} /$ day for an approximate dose of $10 \mathrm{mg} / \mathrm{kg} \mathrm{CNO}$ daily.

\section{Open-Field Locomotion Procedures}

Open-field locomotion assays were performed using a Panasonic WV-CP504 camera with Ethovision XT-9 software (Noldus, Leesburg, VA). Animals were injected intraperitoneally with saline or $1 \mathrm{mg} / \mathrm{kg}$ CNO $35 \mathrm{~min}$ before being placed in the open-field arena for $5 \mathrm{~min}$. On day 1 , animals were injected with saline and allowed to habituate to the testing chamber. Testing occurred on days 2-3, during which animals were injected with saline (day 2) and CNO (day 3). Total distance traveled and time spent in the center of the arena were quantified.

\section{Data Analysis}

Analyses were conducted only in mice with confirmed lesions/AAV expression in the target region. No significant differences were found between litters, so data were pooled across litters. For the lesioning experiments, $t$-tests were used to examine differences between groups. A significant experimental advantage of the DREADDs is that the effects are reversible, enabling a partial within-subjects experimental design in which each animal is tested in both the presence and absence of CNO, and the size of the effect is compared between groups. This partially controls for interanimal variability and enables higher-quality data with a smaller number of animals. For the DREADD ethanol consumption experiments, a difference score between CNO and no $\mathrm{CNO}$ conditions was calculated for each individual mouse, and these difference scores were tested using a one-way ANOVA. Pairwise planned contrasts between groups were performed using the Tukey-Kramer test. In the case of two groups, $t$-tests were used. Corrupted data points due to sipper valves sticking open were omitted from analysis. The same analysis framework was used for consumption of sucrose or water, as well as the open-field locomotion data.

\section{RESULTS}

\section{Electrolytic Lesions of the NAc Core Reduce Ethanol Consumption in the DID Paradigm}

To assess the predictive validity of the DID assay with respect to manipulation of the NAc, we compared animals with electrolytic lesions in the NAc core to sham-lesioned littermates. Lesion placement was confirmed with post hoc immunohistochemistry (Figure 1a). Three separate experiments were performed, with a total of 12 mice: 6 lesioned mice with confirmed lesions confined to the NAc core and 6 sham-lesioned controls. Animals with NAc lesions drank approximately $20 \%$ less ethanol during the 4 -h test session than sham-lesioned littermates (Figure $1 \mathrm{~b} ; t_{(10)}=2.562$,
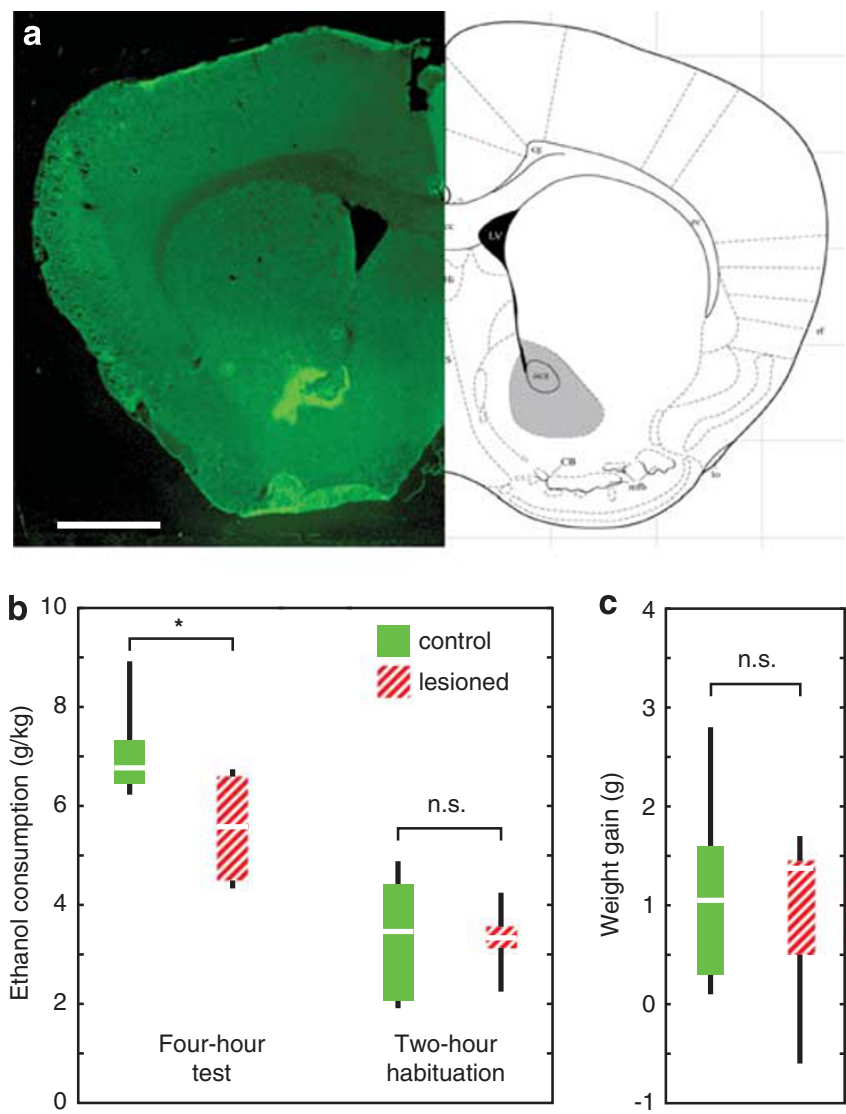

Figure I Electrolytic lesions of the nucleus accumbens core reduce alcohol consumption in the DID paradigm. (a) Electrolytic lesions were localized to the NAc core. Scale bar $=1 \mathrm{~mm}$. Atlas diagram taken from Paxinos and Franklin, AP $=0.98 \mathrm{~mm}$. (b) Mice with lesions in the NAc core consume less ethanol than control animals during the 4-h testing session on day 4. Average consumption during 2-h habituation sessions on days $I-3$ is not different between lesioned and control animals. (c) Weight gain between surgery and the start of the experiment was not different between groups, suggesting that this is not due to a generalized difference in oral intake between groups. $N=6$ lesioned animals, 6 controls. $* P=0.028$, n.s. $=$ not significant.

$P=0.028)$. Interestingly, the lesioned animals did not drink less ethanol during the 2-h habituation sessions (Figure $1 \mathrm{~b}$, $\left.t_{(10)}=0.102, P=0.921\right)$, suggesting that lesions may preferentially affect drinking episodes of long duration or high ethanol consumption. Lesioned and control animals gained the same amount of weight during the 3-week period between surgery and behavioral testing (Figure 1c, $\left.t_{(10)}=0.336, P=0.744\right)$, suggesting that this is not due to a nonspecific reduction in oral intake.

\section{Reverse Pharmacogenetic Inhibition of the NAc Reduces Ethanol Consumption in the DID Paradigm}

To test our hypothesis that reverse pharmacogenetic modulation of the NAc can alter ethanol consumption in the DID paradigm, we used AAV vectors to express the DREADD hM4Di, which inhibits neuronal firing in the presence of $\mathrm{CNO}$, or $\mathrm{hM} 3 \mathrm{Dq}$, which increases neuronal firing rate in the presence of $\mathrm{CNO}$. We used a Credependent FLEX-AAV (Figure 2a), which is inactive until 

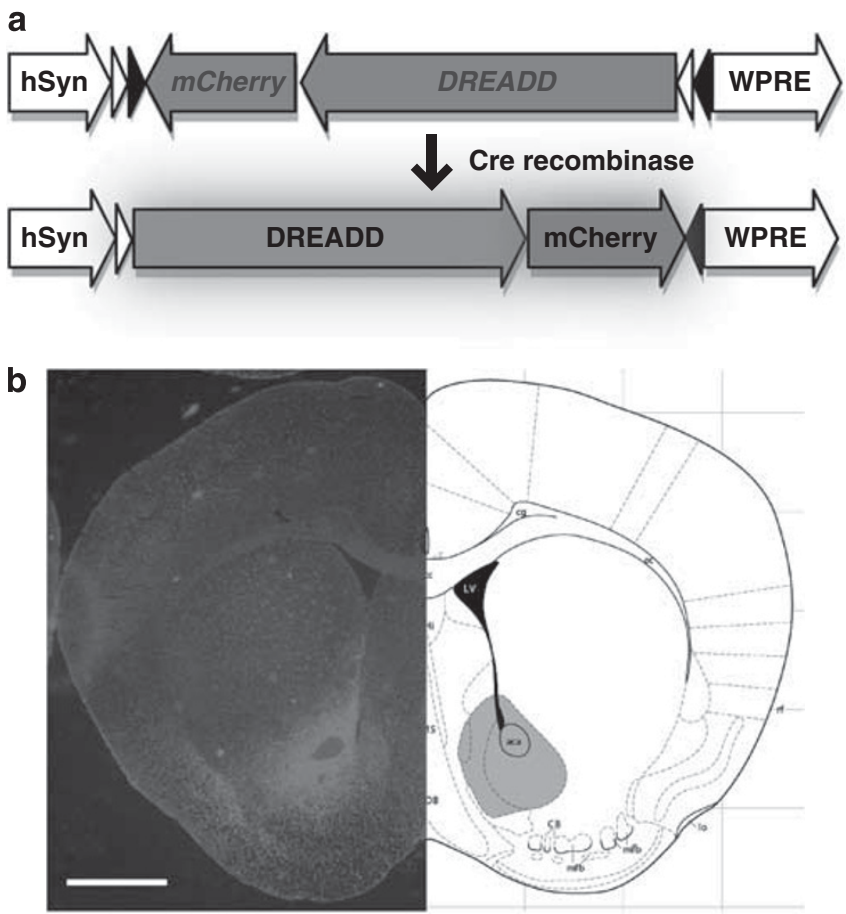

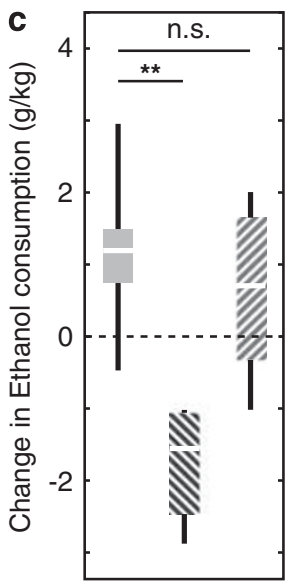

d

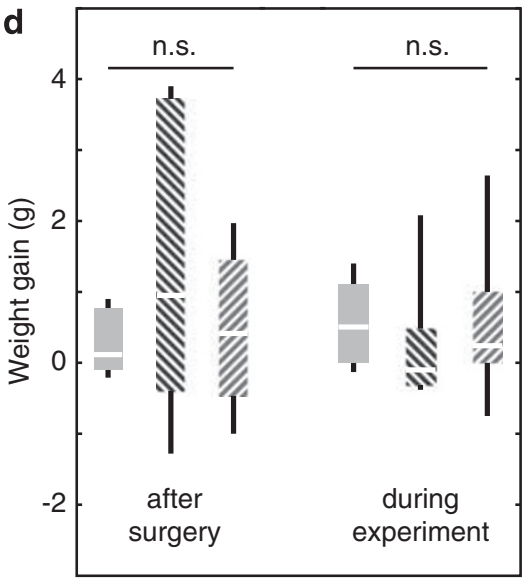

control

N hM4Di
Figure 2 Reverse pharmacogenetic inhibition of the NAc with hM4Di reduces alcohol consumption in the DID paradigm to an extent similar to lesioning. (a) AAV-FLEX-DREADD vectors are inactive until activated by the presence of Cre recombinase. (b) AAV-FLEX-DREADD vectors were coinjected with AAV-Cre vectors, yielding DREADD-mCherry expression in the NAc core and shell. Scale bar $=1 \mathrm{~mm}$. Atlas diagram taken from Paxinos and Franklin, AP $=0.86 \mathrm{~mm}$. (c) Pretreating hM4Di mice with $\mathrm{CNO}$ reduces ethanol consumption relative to baseline and control mice, whereas control and hM3Dq mice are unaffected. (d) There was no between-group difference in weight gain between surgery and the start of the experiment or between the beginning and end of the experiment. $N=6$ controls, 5 hM4Di, 9 hM3Dq. ${ }^{*} * P=0.0006$, n.s. $=$ not significant.

Cre recombinase activates it, initiating expression of the DREADD and mCherry (Krashes et al, 2011). This AAV was coinjected with an AAV of the same serotype expressing Cre, enabling us to express DREADDs nonselectively in all neuronal populations of the NAc (Figure 2b). Control mice were coinjected with AAV-Cre and an AAV encoding only GFP instead of DREADD-mCherry. Four sepa rate experiments were performed, with a total of 20 mice with confirmed GFP/mCherry expression in both the NAc core and shell: 6 GFP control mice, 5 hM4Di mice, and 9 $\mathrm{hM} 3 \mathrm{Dq}$ mice.

As shown in Figure 2c, we found that CNO administration to hM4Di-expressing mice decreased ethanol consumption by $25 \%$, whereas $\mathrm{CNO}$ increased ethanol consumption by $14 \%$ in control mice and $10 \%$ in hM3Dq-expressing mice. This between-group difference was statistically significant $\left(\mathrm{F}_{(2,17)}=12.291, P=0.0005\right)$, and planned contrasts showed that $\mathrm{CNO}$ reduces ethanol consumption in hM4Di-expressing mice relative to controls $\left(q_{(3,17)}=6.579, P=0.0006\right)$, but hM3Dq-expressing mice were not different from controls $\left(q_{(3,17)}=1.367, P=0.607\right)$. An examination of raw behavioral data (Supplementary Figure 3A) showed that GFP control animals did not differ significantly in ethanol consumption from sham-lesioned control animals in the lesioning experiment (Figure $1 \mathrm{~b}, t_{(16)}=1.501, P=0.153$ ). Of note, GFP control animals showed a nonsignificant trend toward increased ethanol consumption after $\mathrm{CNO}$ pretreatment $\left(t_{(5)}=2.437, P=0.059\right)$.

Weight gain by animals was not significantly different between groups for either the period from surgery to start of ethanol access (Figure $2 \mathrm{~d}, \mathrm{~F}_{(2,16)}=0.2, P=0.824$ ) or for the 13-day period from the beginning to the end of ethanol access sessions (Figure $2 \mathrm{~d}, \mathrm{~F}_{(2,16)}=1.08, \quad P=0.363$ ), suggesting that decreased ethanol consumption was not due to a nonspecific effect on oral intake. Variability of weight gain after surgery was somewhat higher in the hM4Di group, but this effect was not observed in either the lesioned animals (Figure 1c) or a subsequent group of hM4Di mice (Supplementary Figure 4), suggesting that this was due to random fluctuation and does not reflect a genuine effect.

\section{Reverse Pharmacogenetic Inhibition of the NAc Does Not Affect Consumption of Sucrose or Water and Does Not Influence Locomotion or Exploratory Behavior}

To explore further whether hM4Di-mediated inhibition of the NAc is specific for drug-related rewards, we tested the effect of NAc inhibition on consumption of sucrose solution and water. Four separate experiments were performed with a total of 32 mice with confirmed GFP/mCherry expression in the NAc: first, eight GFP control mice and eight hM4Di mice were tested for consumption of $5 \%$ sucrose solution and water. Because $5 \%$ sucrose is highly rewarding and may lead to ceiling effects, we also tested consumption of $1 \%$ sucrose in eight GFP control mice and eight hM4Di mice. We performed a modified version of the DID protocol (Supplementary Figure 1C) in which mice had access to either 5\% sucrose solution (days 1-9) and water (days 1015 ), or alternatively $1 \%$ sucrose solution only (days 1-9). We found that there was no between-group difference in consumption of $5 \%$ sucrose (Figure $3 \mathrm{a}, t_{(14)}=0.180$, $P=0.860), 1 \%$ sucrose (Figure $3 \mathrm{~b}, t_{(14)}=0.579, P=0.572$ ), or water (Figure $3 c, t_{(14)}=0.882, P=0.151$ ), suggesting that hM4Di modulation does not decrease consumption of water or sucrose significantly. In GFP control animals, CNO pretreatment increased subsequent consumption of water $\left(t_{(7)}=2.867, \quad P=0.024\right)$ and $1 \%$ sucrose $\left(t_{(7)}=4.442\right.$, $P=0.003)$, but not $5 \%$ sucrose $\left(t_{(7)}=0.031, P=0.976\right)$. 

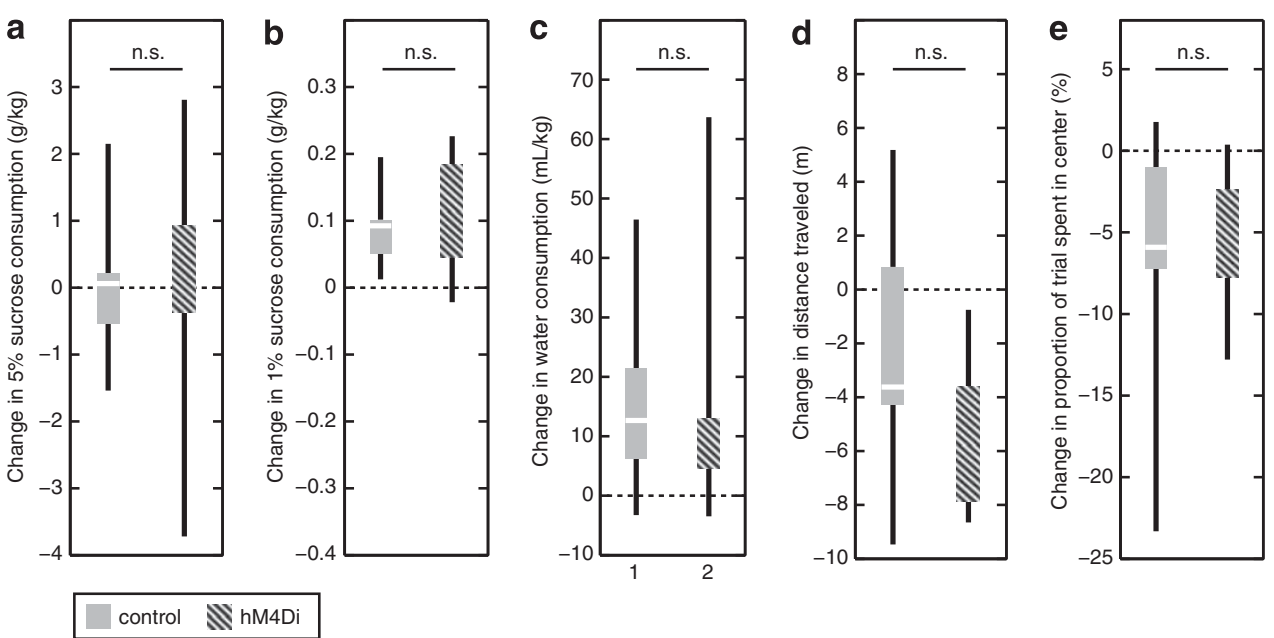

Figure 3 Reverse pharmacogenetic inhibition of the NAc with hM4Di does not affect overall fluid consumption, locomotion, or exploratory behavior. Pretreating hM4Di mice with CNO did not affect (a) 5\% sucrose consumption, (b) 1\% sucrose consumption, or (c) water relative to control mice. Pretreatment of hM4Di mice with CNO also did not affect (d) distance traveled in a 5-min open-field assay or (e) the proportion of time spent exploring the center of an open arena relative to controls. $N=8$ controls, 8 hM4Di. n.s. $=$ not significant.

In tests of open-field locomotion, we found that the change in distance traveled after $\mathrm{CNO}$ administration was not significantly different between groups (Figure 3d, $\left.t_{(13)}=1.738, P=0.106\right)$, suggesting that hM4Di modulation does not affect general locomotion. The change in amount of time spent in the center of the arena after CNO administration was also not significantly different between groups (Figure $3 e, t_{(13)}=0.408, P=0.690$ ), suggesting that hM4Di modulation does not affect exploratory activity. Although there was no difference between groups, both showed statistically significant decreases in distance traveled $\left(t_{(14)}=3.797, P=0.002\right)$ and center time $\left(t_{(14)}=3.543\right.$, $P=0.003)$ after $\mathrm{CNO}$ administration. These decreases were statistically indistinguishable from the decrease between the first and second saline injection sessions $\left(t_{(14)}=1.722\right.$, $P=0.107$ for distance traveled, $t_{(14)}=1.279, P=0.222$ for center time; data not shown), suggesting that the decreased locomotion and exploration after $\mathrm{CNO}$ administration is due to habituation to the chamber rather than an effect of the drug. As in our ethanol consumption experiments, we saw no difference between groups in weight gain for either the period from surgery to start of the experiment (Supplementary Figure $4, t(14)=0.749, P=0.467$ ) or from the start of the experiment to the end (Supplementary Figure $4, t(14)=1.804, P=0.093)$.

\section{DISCUSSION}

In this study, we found that electrolytic lesions of the NAc core reduce ethanol consumption in the DID limited access paradigm in C57BL/6J mice. Interestingly, we only saw this effect during the 4-h test session and not during the 2-h habituation sessions, suggesting that lesions of the NAc core decrease ethanol consumption preferentially under conditions of the longest duration or highest intake. We also found that inhibiting activity in the NAc with hM4Di decreased ethanol consumption relative to controls, whereas increasing activity with $\mathrm{hM} 3 \mathrm{Dq}$ had no effect.
hM4Di modulation appeared to affect ethanol consumption preferentially and did not affect water consumption, sucrose consumption, or open-field locomotion or exploration. Surprisingly, we found that in control mice, CNO pretreatment increased consumption of water and $1 \%$ sucrose, with a strong trend toward increased consumption of ethanol, but no indication of increased consumption of 5\% sucrose. As CNO is believed to be pharmacologically inert in mice, the most likely explanation is that the mice could taste the $\mathrm{CNO}$ in their drinking water, and its removal improved the flavor of subsequently consumed liquids. The absence of increased 5\% sucrose consumption likely represents a ceiling effect in which a sufficiently strong taste-based reward drove animals to consume maximal amounts of liquid (Supplementary Figure 3B), obscuring the much smaller effect of CNO removal. Whether or not this explanation is correct will be an interesting topic for future studies, but ultimately this nonspecific effect does not affect our current results because our experimental design accounts for it by comparing DREADD mice to GFPexpressing controls.

\section{The Effects of NAc Core Lesions Support the Predictive Validity of the DID Model}

DID and other similar limited access ethanol consumption paradigms are relatively high-throughput models that exhibit good predictive validity for medications currently used for the treatment of alcohol dependence, such as naltrexone, baclofen, and mGluR5 antagonists such as acamprosate (Tanchuck et al, 2011). Our results support the predictive validity of the DID model with respect to manipulations of the NAc core, which is the primary target for neurosurgical interventions for alcohol dependence and other addictive disorders in humans. Previous studies in rodent models present a somewhat complex picture regarding the effects of NAc lesions on ethanol consumption. Studies by Hansen et al have shown that axon-sparing ibotenic acid lesions of the NAc increase ethanol consumption 
in a free access paradigm (Hansen et al, 1995; Johansson and Hansen, 2000). However, animals in free access paradigms have large fluctuations in blood ethanol concentration that only rarely reach pharmacologically significant levels, raising questions about the validity of these models for studying addictive behavior (Dole and Gentry, 1984). A more recent study by Dhaher et al (2009) using electrolytic lesions of the medial NAc shell compared 24-h free access to a 2-h limited access paradigm, finding that lesioned animals had a nonsignificant trend toward increased consumption in free access but a significant decrease in consumption in the limited access paradigm. Our results differ slightly from Dhaher et al in that we saw no difference in ethanol consumption in 2-h limited access, but we did see a significant decrease in ethanol consumption during 4-h limited access. This difference is likely due to the fact that our experiments lesioned the NAc core, similar to human studies, whereas Dhaher et al lesioned the medial NAc shell. This is consistent with prior lesion studies demonstrating that both the core and shell both have important, but distinct, roles in alcohol-related behaviors (Chaudhri et al, 2010; Gremel and Cunningham, 2008). In aggregate, these results support the notion that limited access ethanol consumption paradigms such as DID may be useful preclinical model systems for understanding and improving NAc manipulations for the clinical treatment of alcohol abuse and dependence. The precision and generalizability of DID can also be improved beyond the protocol used in our study by measuring blood ethanol concentrations. However, we elected not to perform these because ethanol consumption is strongly predictive of blood concentration in C57BL/6 $\mathrm{J}$ mice (Rhodes et al, 2005), and because in our hands serial blood collection altered ethanol consumption on subsequent days.

\section{Reverse Pharmacogenetic Modulation of the NAc}

Our results with hM4Di suggest that reverse pharmacogenetic inhibition of activity in the NAc preferentially suppresses consumption of ethanol over natural rewards. However, increasing firing in the NAc with hM3Dq had no effect on ethanol consumption. The simplest explanation for this asymmetry would be that the cells in the NAc that drive consumption behavior have high firing rates during limited access ethanol consumption; decreasing firing rates would thus decrease consumption, but increasing them further would have no effect. Confirmation of this hypothesis will require future studies combining reverse pharmacogenetic modulation with extracellular recording of neuronal activity in the NAc during ethanol consumption.

Although our results with hM4Di represent the first demonstration that reverse pharmacogenetic activation of $\mathrm{G}_{\mathrm{i} / \mathrm{o}}$ signaling in the NAc reduces ethanol consumption, there have been prior studies examining the effect of viral vector-mediated expression of $\mathrm{G}_{\mathrm{i} / \mathrm{o}}$-coupled neurotransmitter receptors in the NAc. A series of studies by Thanos et al found that overexpression of the D2 dopamine receptor, which is $\mathrm{G}_{\mathrm{i} / \mathrm{o}}$ coupled, in the NAc reduces ethanol consumption in both rats and mice in a variety of different conditions (Thanos et al, 2005; Thanos et al, 2004; Thanos et al, 2001). However, two studies from Hoplight $e t$ al that overexpress the $5-\mathrm{HT}_{1 B}$ receptor, which is also $\mathrm{G}_{\mathrm{i} / \mathrm{o}}$ coupled, in the NAc showed the opposite result of significant increases in ethanol consumption under similar conditions (Furay et al, 2011; Hoplight et al, 2006). One possible explanation for this discrepancy is that we used AAV5, whereas Thanos et al used adenovirus and Hoplight et al used herpes simplex virus. Differences in viral tropism could lead to preferential receptor expression in different NAc neuronal subtypes, altering activity in the circuit in different ways. Another possible explanation is that the time-varying concentrations of the endogenous ligands for the $\mathrm{D} 2$ and $5-\mathrm{HT}_{1 \mathrm{~B}}$ receptors may differ significantly in ways that could be difficult to predict, which illustrates an advantage of the reverse pharmacogenetic approach in unambiguously determining the role of specific signaling pathways in behavior.

\section{Future Clinical Prospects of Reverse Pharmacogenetics}

In experimental animals, the primary drawback of reverse pharmacogenetic methods is the relatively slow temporal resolution, which is limited by the pharmacokinetics of drug delivery and clearance. Optogenetic methods overcome this limitation by replacing the systemically administered drug with focally administered light pulses, enabling rapid temporal control of activity (Fenno et al, 2011; Sjulson and Miesenböck, 2008). For clinical applications, however, optogenetics is hampered by several practical considerations. Notably, it requires ectopic expression of high levels of microbial proteins, which creates the risk of an immune reaction, and it must also overcome the limited propagation of light through brain tissue, which necessitates invasive implantation of indwelling optical fibers and is unlikely to scale well from the small brains of mice to the large brains of humans. For most clinical applications, the low temporal resolution of reverse pharmacogenetic methods is a much less severe constraint. Although DREADDs and CNO are not currently usable clinically, AAV vectors have been used successfully in multiple clinical trials to express transgenes in the human brain (Christine et al, 2009; LeWitt et al, 2011; Souweidane et al, 2010), and the number of technical issues preventing clinical application of reverse pharmacogenetics is substantially smaller than for optogenetics. The primary technical issues remaining include (1) finding receptor-ligand pairs that can serve as human-optimized alternatives to the DREADD-CNO pair, (2) targeting AAV to specific cell types in a human brain (ie, without using transgenic Cre lines), and (3) developing a safety mechanism by which transgene expression can be inactivated permanently if adverse outcomes occur. These problems are nontrivial, but they are well circumscribed, technically tractable, and likely to be solved in the near future. The greater challenge will be to find and validate specific targets for modulation that are likely to have a therapeutic effect in the treatment of addiction. Our results indicate that reverse pharmacogenetic modulation of the NAc selectively reduces alcohol consumption in a preclinical model to an extent similar to irreversible lesioning, providing support for reverse pharmacogenetics as a future therapeutic strategy and for the NAc as a potential therapeutic target. 


\section{FUNDING AND DISCLOSURE}

Dr Jared Van Snellenberg has received compensation as a consultant for Monclarity, LLC. The remaining authors declare no conflict of interest.

\section{ACKNOWLEDGEMENTS}

We thank Michael Krashes, Brad Lowell, Bryan Roth, and Jürgen Wess for sharing DREADD constructs and reagents. CNO was obtained from the NIH as part of the Rapid Access to Investigative Drug Program funded by the NINDS. We also thank Michael Long and Dmitriy Aronov for advice on electrolytic lesions, Charles Hoeffer and the NYU rodent behavior core facility for help with behavioral assays, and Jens Hjerling-Leffler for valuable comments on the manuscript. This work was supported by funds from the NYU Physician Scientist Training Program (LS); the NYU Dean's Undergraduate Research Fund (DC); NIH grants R01 MH068469, R01 MH071679, R01 MH095147, and R01 NS081297 to GF; and grant UL1 TR000038 from the National Center for Advancing Translational Sciences, National Institutes of Health.

\section{REFERENCES}

Alexander GM, Rogan SC, Abbas AI, Armbruster BN, Pei Y, Allen JA et al (2009). Remote control of neuronal activity in transgenic mice expressing evolved G protein-coupled receptors. Neuron 63: 27-39.

Armbruster BN, Li X, Pausch MH, Herlitze S, Roth BL (2007). Evolving the lock to fit the key to create a family of G proteincoupled receptors potently activated by an inert ligand. Proc Natl Acad Sci USA 104: 5163-5168.

Bouchery EE, Harwood HJ, Sacks JJ, Simon CJ, Brewer RD (2011). Economic costs of excessive alcohol consumption in the U.S., 2006. Am J Prev Med 41: 516-524.

CDC (2008, Alcohol Related Disease Impact (ARDI) application. Available at http://apps.nccd.cdc.gov/DACH_ARDI/Default.aspx.

Chang WH, Lin SK, Lane HY, Wei FC, Hu WH, Lam YW et al (1998). Reversible metabolism of clozapine and clozapine N-oxide in schizophrenic patients. Prog Neuro-psychopharmacol Biol Psychiatry 22: 723-739.

Chaudhri N, Sahuque LL, Schairer WW, Janak PH (2010). Separable roles of the nucleus accumbens core and shell in context- and cue-induced alcohol-seeking. Neuropsychopharmacology 35: 783-791.

Christine CW, Starr PA, Larson PS, Eberling JL, Jagust WJ, Hawkins RA et al (2009). Safety and tolerability of putaminal AADC gene therapy for Parkinson disease. Neurology 73: 16621669.

Dhaher R, Finn DA, Oberbeck DL, Yoneyama N, Snelling CC, Wu $\mathrm{W}$ et al (2009). Electrolytic lesions of the medial nucleus accumbens shell selectively decrease ethanol consumption without altering preference in a limited access procedure in C57BL/6J mice. Pharmacol Biochem Behav 92: 335-342.

Dole VP, Gentry RT (1984). Toward an analogue of alcoholism in mice: scale factors in the model. Proc Natl Acad Sci USA 81: 3543-3546.

Fenno L, Yizhar O, Deisseroth K (2011). The development and application of optogenetics. Annu Rev Neurosci 34: 389-412.

Furay AR, Neumaier JF, Mullenix AT, Kaiyala KK, Sandygren NK, Hoplight BJ (2011). Overexpression of 5-HT(1B) mRNA in nucleus accumbens shell projection neurons differentially affects microarchitecture of initiation and maintenance of ethanol consumption. Alcohol 45: 19-32.

Gremel CM, Cunningham CL (2008). Roles of the nucleus accumbens and amygdala in the acquisition and expression of ethanol-conditioned behavior in mice. J Neurosci 28: 1076-1084.

Hansen S, Fahlke C, Hard E, Thomasson R (1995). Effects of ibotenic acid lesions of the ventral striatum and the medial prefrontal cortex on ethanol consumption in the rat. Alcohol 12: 397-402.

He F, Guan H, Zhao Z, Miao X, Zhou Q, Li L et al (2008). Evaluation of short-term psychological functions in opiate addicts after ablating the nucleus accumbens via stereotactic surgery. Stereotact Funct Neurosurg 86: 320-329.

Heinz A, Beck A, Grusser SM, Grace AA, Wrase J (2009). Identifying the neural circuitry of alcohol craving and relapse vulnerability. Addict Biol 14: 108-118.

Heinze HJ, Heldmann M, Voges J, Hinrichs H, Marco-Pallares J, Hopf JM et al (2009). Counteracting incentive sensitization in severe alcohol dependence using deep brain stimulation of the nucleus accumbens: clinical and basic science aspects. Front Hum Neurosci 3: 22.

Hoplight BJ, Sandygren NA, Neumaier JF (2006). Increased expression of 5-HT1B receptors in rat nucleus accumbens via virally mediated gene transfer increases voluntary alcohol consumption. Alcohol 38: 73-79.

Johansson AK, Hansen S (2000). Increased alcohol intake and behavioral disinhibition in rats with ventral striatal neuron loss. Physiol Behav 70: 453-463.

Koob GF, Volkow ND (2010). Neurocircuitry of addiction. Neuropsychopharmacology 35: 217-238.

Krashes MJ, Koda S, Ye C, Rogan SC, Adams AC, Cusher DS et al (2011). Rapid, reversible activation of AgRP neurons drives feeding behavior in mice. J Clin Invest 121: 1424-1428.

Kuhn J, Grundler TO, Bauer R, Huff W, Fischer AG, Lenartz D et al (2011). Successful deep brain stimulation of the nucleus accumbens in severe alcohol dependence is associated with changed performance monitoring. Addict Biol 16: 620-623.

LeWitt PA, Rezai AR, Leehey MA, Ojemann SG, Flaherty AW, Eskandar EN et al (2011). AAV2-GAD gene therapy for advanced Parkinson's disease: a double-blind, sham-surgery controlled, randomised trial. Lancet Neurol 10: 309-319.

Lobo MK, Nestler EJ (2011). The striatal balancing act in drug addiction: distinct roles of direct and indirect pathway medium spiny neurons. Front Neuroanat 5: 41.

Ray RS, Corcoran AE, Brust RD, Kim JC, Richerson GB, Nattie E et al (2011). Impaired respiratory and body temperature control upon acute serotonergic neuron inhibition. Science 333: 637-642.

Rhodes JS, Best K, Belknap JK, Finn DA, Crabbe JC (2005). Evaluation of a simple model of ethanol drinking to intoxication in C57BL/6J mice. Physiol Behav 84: 53-63.

Rodgers DA, McClearn G (1962). Mouse strain differences in preference for various concentrations of alcohol. Q J Stud Alcohol 23: 26-33.

Rogan SC, Roth BL (2011). Remote control of neuronal signaling. Pharmacol Rev 63: 291-315.

Sjulson L, Miesenböck G (2008). Photocontrol of neural activity: biophysical mechanisms and performance in vivo. Chem Rev 108: 1588-1602.

Souweidane MM, Fraser JF, Arkin LM, Sondhi D, Hackett NR, Kaminsky SM et al (2010). Gene therapy for late infantile neuronal ceroid lipofuscinosis: neurosurgical considerations. J Neurosurg Pediatr 6: 115-122.

Tanchuck MA, Yoneyama N, Ford MM, Fretwell AM, Finn DA (2011). Assessment of GABA-B, metabotropic glutamate, and opioid receptor involvement in an animal model of binge drinking. Alcohol 45: 33-44. 
Tepper JM, Tecuapetla F, Koos T, Ibanez-Sandoval O (2010). Heterogeneity and diversity of striatal GABAergic interneurons. Front Neuroanat 4: 150.

Thanos PK, Rivera SN, Weaver K, Grandy DK, Rubinstein M, Umegaki $\mathrm{H}$ et al (2005). Dopamine D2R DNA transfer in dopamine D2 receptor-deficient mice: effects on ethanol drinking. Life Sci 77: 130-139.

Thanos PK, Taintor NB, Rivera SN, Umegaki H, Ikari H, Roth G et al (2004). DRD2 gene transfer into the nucleus accumbens core of the alcohol preferring and nonpreferring rats attenuates alcohol drinking. Alcohol Clin Exp Res 28: 720-728.
Thanos PK, Volkow ND, Freimuth P, Umegaki H, Ikari H, Roth $\mathrm{G}$ et al (2001). Overexpression of dopamine D2 receptors reduces alcohol self-administration. $J$ Neurochem 78: 1094-1103.

Voges J, Muller U, Bogerts B, Munte T, Heinze HJ (2012). Deep brain stimulation surgery for alcohol addiction. World Neurosurg 80: e21-S28.e31.

Wu HM, Wang XL, Chang CW, Li N, Gao L, Geng N et al (2010). Preliminary findings in ablating the nucleus accumbens using stereotactic surgery for alleviating psychological dependence on alcohol. Neurosci Lett 473: 77-81.

Supplementary Information accompanies the paper on the Neuropsychopharmacology website (http://www.nature.com/npp) 